\title{
The numerical study of the influence of material parameters on the regime of adhesive wear of surface asperities
}

\author{
Ivan Dudkin ${ }^{1, *}$, Evgeny Shilko ${ }^{1,2}$, Andrey Dimaki ${ }^{1}$, and Valentin Popov ${ }^{3}$ \\ ${ }^{1}$ Institute of Strength Physics and Materials Science SB RAS, 634055, pr. Akademicheskii 2/4., \\ Tomsk, Russia \\ ${ }^{2}$ Tomsk State University, Lenin ave. 36, Tomsk 634050, Russia \\ ${ }^{3}$ Berlin University of Technology, str. des 17 Juni 135, Berlin 10623, Germany
}

\begin{abstract}
In the paper, we developed a discrete element model of adhesive interaction of materials with taking into account the "cold welding" effect. Based on this model, we carried out a detailed analysis of the factors controlling regimes of adhesive wear for elastic-plastic materials with nonideal plasticity. We proposed an empirical criterion of transition from "breakaway" to "grinding" regime of wear. The extended form of the given criterion takes into account the dependence of material strength on hydrostatic pressure.
\end{abstract}

\section{Introduction}

Wear of surfaces under the condition of tangential contact occurs due to the presence of asperities of various scales. The wear rate is determined by a number of factors [1-5]. Among them, the key role belongs to the formation of a bond between the contact surfaces (adhesion) in addition to the determining influence of the geometry of asperities and the conditions of their local contact [6]. Adhesive interaction stimulates both plastic strain localization in the spot of local contact and tearing out of wear particles. The role of adhesive interaction increases many times in the case of friction of clean surfaces with high surface energy since in this case, their "cold welding" is possible $[7,8]$.

Despite a large number of research works, the conditions and mechanisms for the formation of individual wear particles and wear debris layer are still widely debatable, although they have been studied experimentally for many years. Currently used wear laws are limited only to fully or semi-empirical formulas, which have a strongly limited predictive ability [9]. Understanding the regularities of the elementary acts of the process of adhesive wear and the nonlinear effect of adhesion force on the wear mode and regime is extremely important for a reliable prediction of the intensity of wear $[10,11]$. The key to this understanding is analytical and numerical studies aimed to reveal these regularities for single asperities or their ensembles. In particular, Rabinovich proposed the concept of wear, in which the separation of debris from the surface, their adhesion to the opposite surface

\footnotetext{
* Corresponding author: workdudkin@gmail.com
} 
and so on (up to the formation of fully loose particles) takes place [12-14]. The limitations of this concept are associated with the neglect of plastic deformation as a wear mechanism. In contrast, Holm's concept [15] is based on the fact that plastic wear of asperities is the leading mechanism of wear. In a recent numerical molecular dynamic study [16], an attempt was made to combine these concepts. In particular, it was shown that adhesive wear can develop in two ways: 1) formation and growth of wear particles or 2) plastic deformation of nano- and microcontacts. However, this and many other works do not fully answer the question about the generality of the obtained regularities and the influence of the magnitude of the macroscopic characteristics of material on the conditions for the implementation of a particular wear mode.

These unsolved problems determine the ever-increasing interest in studying the factors that control adhesive wear. This paper is devoted to the study of the implementation conditions of different regimes of adhesive wear of single asperities by means of computer modeling. We analyze the influence of the material parameters determining the plasticity and strength on the condition of wear rate change from low-wear to the high-wear regime.

\section{Problem statement}

We carried out the research with the use of the method of movable cellular automata (MCA) described in a number of recent papers [17-20]. The MCA is one of the advanced implementations of a discrete element (DE) approach. Its formalism is based on an approximation of homogeneously deformable discrete elements and many-body formulation of the relations for element-element interaction forces.

In the paper, we study the contact interaction of isotropic linearly elastic and elasticplastic materials. In the latter case, we consider the materials having the macroscopically inelastic response caused by the evolution of the defects on the crystal lattice scale. Modeling of the mechanical behavior of materials in the range of elastic response (below the yield stress) is performed using Hooke's law:

$$
\boldsymbol{\sigma}_{\alpha \beta}=2 G \boldsymbol{\varepsilon}_{\alpha \beta}+(1-2 G / K) \sigma_{\text {mean }} \delta_{\alpha \beta},
$$

where $\alpha, \beta=\mathrm{x}, \mathrm{y}, \mathrm{z} ; G$ and $K$ are the shear and bulk elastic moduli of the material; $\sigma_{\text {mean }}$ is the mean stress; $\delta_{\alpha \beta}$ is the Kronecker delta. The behavior of the material beyond the yield stress was simulated using the associated plastic flow model with von Mises yield criterion:

$$
\sigma_{\text {eq }}=\sigma_{\mathrm{y}},
$$

where $\sigma_{\mathrm{eq}}$ is equivalent stress, $\sigma_{\mathrm{y}}$ is the yield strength of the material.

Local fracture in DEM is modeled by means of change of the state of the pair of discrete elements (transition from "linked" state of the pair to "unlinked" state). This transition is governed by the assigned fracture criterion for the pair. In the study we used twoparametric failure criterion of Drucker and Prager in the following form:

$$
\sigma_{\text {eq }} 0.5(a+1)+\sigma_{\text {mean }} 1.5(a-1)=\sigma_{\mathrm{c}},
$$

where $a=\sigma_{\mathrm{c}} / \sigma_{\mathrm{t}}, \sigma_{\mathrm{c}}$ is the uniaxial compressive strength of the material, $\sigma_{\mathrm{t}}$ is the uniaxial tensile strength. These two uniaxial strengths of the material are input parameters of the fracture criterion. Note that the value of shear strength $\sigma_{\mathrm{j}}$ of the material can be easily derived from this criterion $\left(\sigma_{\mathrm{eq}}=\sqrt{3} \sigma_{\mathrm{j}}, \sigma_{\text {mean }}=0\right)$ : 


$$
\sigma_{\mathrm{j}}=\frac{2 \sigma_{\mathrm{c}} \sigma_{\mathrm{t}}}{\sqrt{3}\left(\sigma_{\mathrm{c}}+\sigma_{\mathrm{t}}\right)} .
$$

Von Mises failure criterion $\sigma_{\text {eq }}=\sigma_{\mathrm{t}}$, which is often applied to highly ductile materials, is a partial case (at $a=1$ ) of the two-parametric Drucker-Prager fracture criterion.

In order to simulate an adhesive interaction between discrete elements, Dugdale's model of the cohesive zone was used [21]. This model postulates a constant value of adhesion stress $\sigma_{D}$ between two interacting surfaces if a separation between these surfaces doesn't exceed a certain distance $l_{\text {adh }}$ that can be interpreted as a "length" of the adhesive potential. The work of adhesion (surface energy) $\Delta \gamma$ within the Dugdale's model is calculated by the formula: $\Delta \gamma=\sigma_{\mathrm{D}} l_{\text {adh }}$. The adhesive force acts between unlinked and uncontact elements and leads to their mutual attraction as well as to their straining (elongation along the line connecting mass centers of the elements).

In the study, we considered the "limiting" case of non-oxidized (chemically clean) and atomically smooth surfaces of the discrete elements. In the framework of this assumption, we assumed the possibility of "cold welding" of the surfaces under contact. In other words, under direct mechanical contact of the surfaces, a chemical bonding occurs between them (an unlinked pair of model discrete elements becomes linked). Following this assumption, we used the Drucker-Prager fracture criterion as a condition of detachment (destruction of a chemical bond) of a newly linked pair of discrete elements. The parameters of the fracture criterion are the same as for intact material.

\section{Numerical analysis of the regimes of wear}

Using the developed DEM-based model, we numerically studied the adhesive contact interaction between surface asperities of interacting bodies in the 2D problem statement and plane strain approximation. Figure 1 shows the structure and the loading scheme of the model 2D sample. Interacting asperities have the same size and trapezoidal shape. These contact asperities have a height equal to $D=0.5 \mathrm{~mm}$, the size of the discrete element is $d=0.05 \mathrm{~mm}$.

We analyzed the interaction of these asperities under the condition of relative tangential motion (sliding) of the surfaces with constant velocity. This regime of sliding was realized by fixing the vertical positions of the upper and the lower faces of the sample and applying constant horizontal velocities $V$ to these faces in opposite directions. Periodic boundary conditions in the horizontal direction were specified on the lateral faces of the sample.

The materials of the contacting bodies were the same and had elastic-plastic properties. We specified the following mechanical characteristics of the model material of the contacting bodies: Young's modulus $E=1 \mathrm{GPa}$, density $\rho=2000 \mathrm{~kg} / \mathrm{m}^{3}$, Poisson's ratio $v=0.3$, linear strain hardening with a coefficient $H=0.5 \mathrm{GPa}$, yield stress $\sigma_{\mathrm{y}}=1 \mathrm{MPa}$, $\sigma_{\mathrm{c}}=\sigma_{\mathrm{t}}=5 \mathrm{MPa}(a=1)$. These values are reminiscent of the values of the mechanical parameters of some polymers with elastic-plastic rheological properties. 


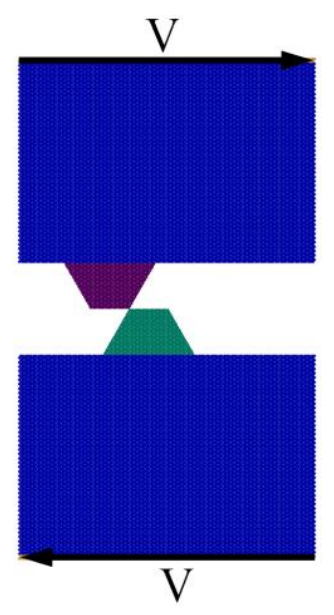

Fig. 1. The structure of the model sample and the scheme of loading

In the study, we assumed atomic smoothness of surfaces of contacting discrete elements. In this case, the value of the parameter $l_{\text {adh }}$ of Dugdale's model of adhesive interaction should be of the atomic scale. We used reasonable value $l_{\mathrm{adh}}=1 \mathrm{~nm}$, which is an upper bound of the characteristic distance of adhesive interaction between atomic planes.

We investigated the influence of mechanical parameters of material (including strength ratio $a$, shear strength, yield stress, and strain hardening coefficient) on the regime of wear of interacting asperities. Such an analysis was carried out by varying the value of each mechanical parameter relative to the above mentioned "reference" value, leaving the other parameters unchanged. For each given set of these mechanical parameters, a series of numerical simulations were conducted with different values of Dugdale's adhesion stress $\sigma_{\mathrm{D}}$. We varied adhesion stress from 0 up to the upper physical limit that is a tensile strength $\sigma_{\mathrm{t}}$. Based on the results of these simulations we determined the intervals of $\sigma_{\mathrm{D}}$ corresponding to different regimes of wear ("breakaway" and "grinding", which are discussed further) and the "boundary" (threshold) value $\sigma_{\mathrm{D}}^{*}$ of the adhesion stress.

We found that two wear regimes may occur according to the relation of an asperity size and characteristic length. In the first one that we may refer to as a "breakaway" regime, asperities separate from the contacting bodies as a whole (see Figure 2). According to the criterion of Rabinowicz [12], this effect takes place when asperity size exceeds a critical size. 

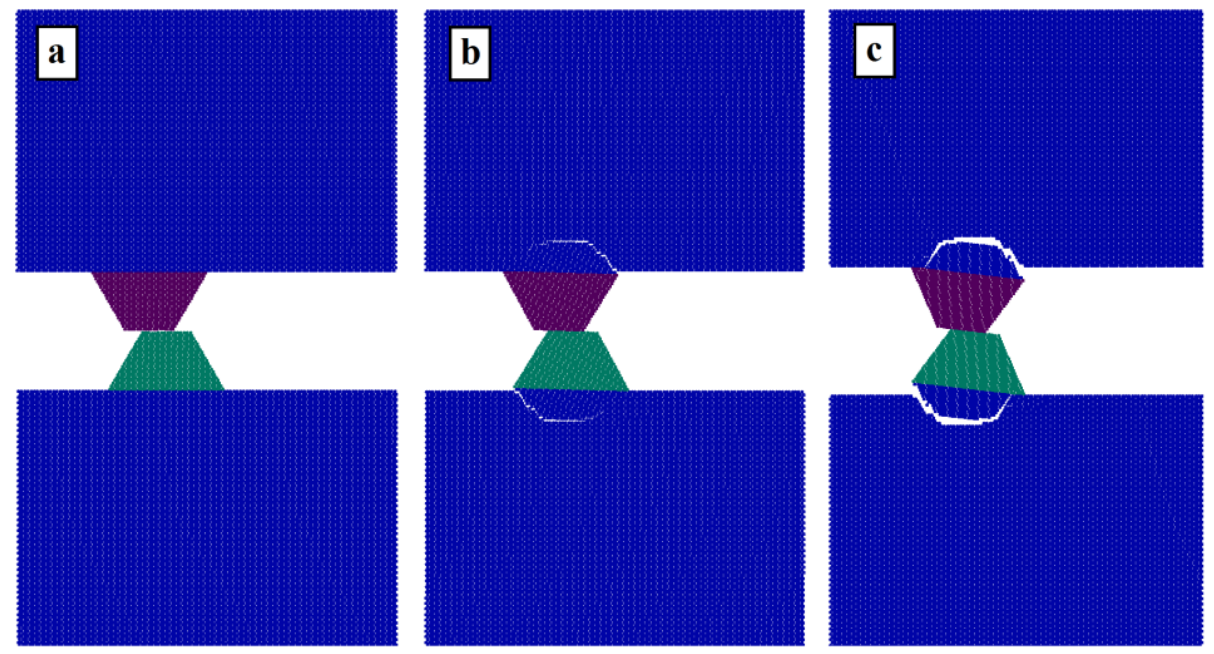

Fig. 2. Evolution of the asperities detachment in the "breakaway" regime of wear

In the second regime called "grinding" asperities are being gradually destroyed within contact patches during the relative motion (see Figure 3). In the grinding regime, a detachment of "large" (compared to asperity size) fragments of asperities doesn't take place. According to the criterion of Rabinowicz, the given regime occurs when asperity size is smaller than some critical size determined by material parameters.
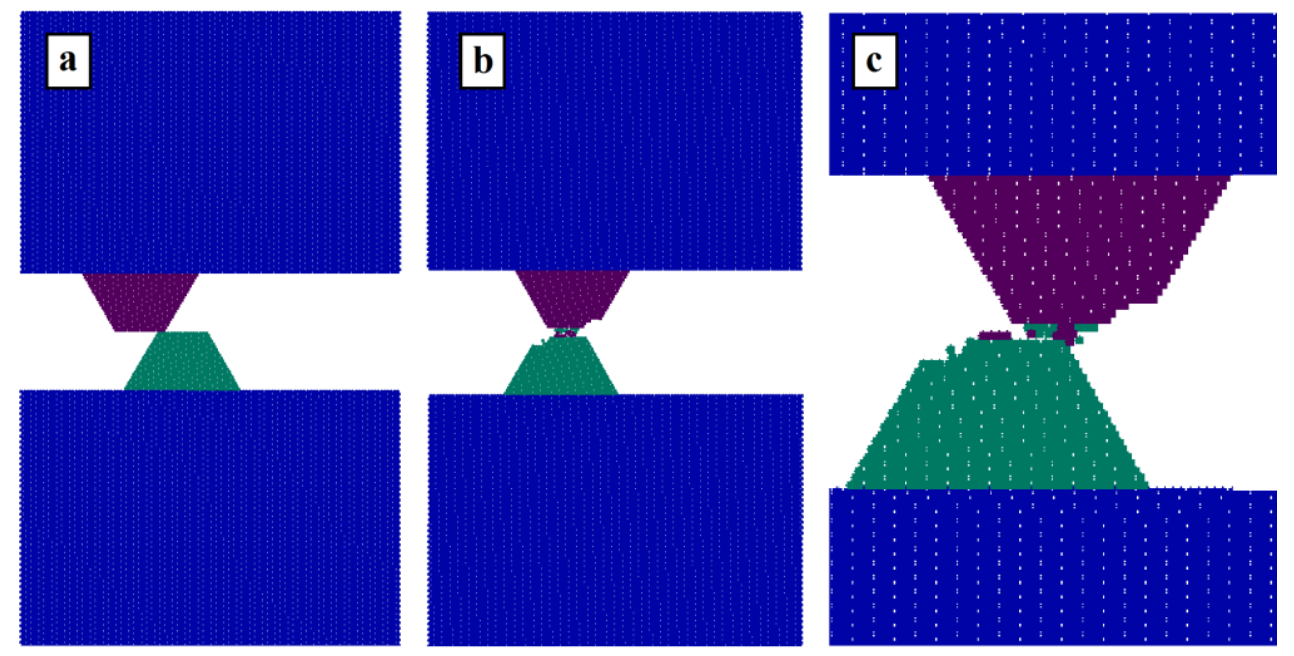

Fig. 3. "Grinding” regime of asperities destruction

When asperity size is close to the critical size, an intermediate regime of destruction called "cleavage" takes place (see Figure 4). It is necessary to note that the transition to "grinding" regime of destruction takes place only if the adhesive force $\sigma_{\mathrm{D}}$ exceeds some critical value $\sigma_{\mathrm{D}}^{*}$. At that, the latter condition is necessary but not sufficient: under some 
combinations of governing parameters and $\sigma_{\mathrm{D}} \leq \sigma_{\mathrm{t}}$ the wear regime tends to "breakaway" or "cleavage" mode.
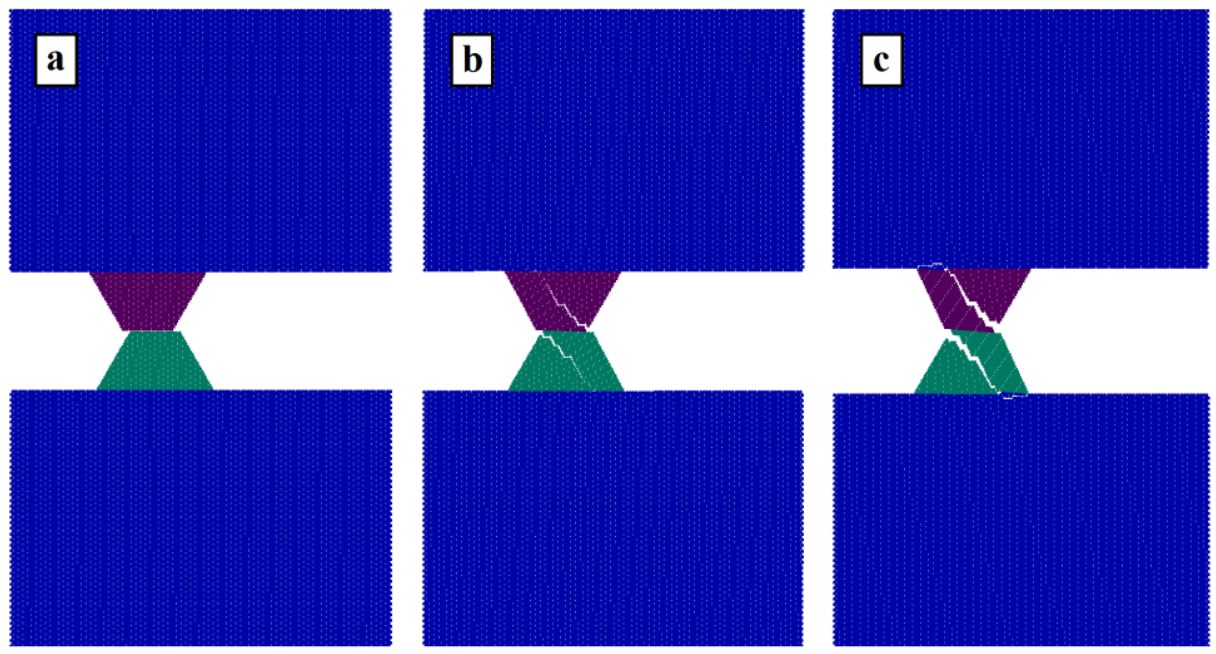

Fig. 4. "Cleavage" regime of asperities destruction

\section{Discussion}

The results of numerical simulation have shown that the values of the material parameters, in particular, the value of the material strength, determine the threshold value $\sigma_{\mathrm{D}}^{*}$. In order to study the mentioned phenomenon in a more detail, we have carried out a series of numerical calculations with use of the initially set ("reference") values of mechanical parameters of the material $\left(\rho, E, v, \sigma_{\mathrm{y}}, H, a=1\right)$ but different values of tensile strength $\sigma_{\mathrm{t}}$ (the only parameter of von Mises fracture criterion). For each value of von Mises strength, we varied the value of specific adhesive force $\sigma_{\mathrm{D}}$ from 0 up to $\sigma_{\mathrm{t}}$. The "cloud" of points corresponding to calculations with different values of von Mises strength and specific adhesive force is shown in Figure 5. Note that shear strength $\sigma_{\mathrm{j}}=\sigma_{\mathrm{t}} / \sqrt{3}$ is used as an argument in the plot instead of the tensile strength $\sigma_{\mathrm{t}}$. The border values of $\sigma_{\mathrm{D}}$ separating the regions of "breakaway" and "grinding" regimes of wear are threshold adhesion stresses $\sigma_{\mathrm{D}}^{*}$. It is clearly seen that threshold adhesion stress depends on material shear strength, and the border curve separating two different regions of wear is well approximated by the straight line $\sigma_{\mathrm{D}}^{*}=A^{*} \sigma_{\mathrm{j}}$. 


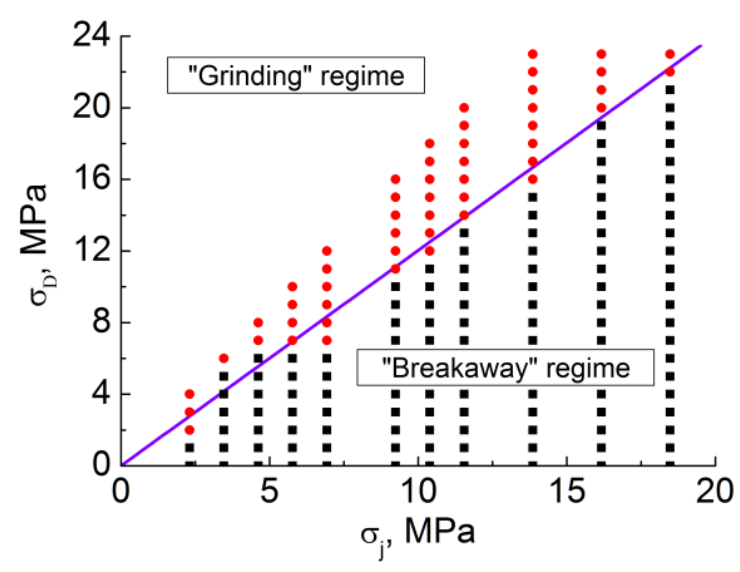

Fig. 5. The "cloud" of points in the coordinate plane $\left(\sigma_{\mathrm{j}}, \sigma_{\mathrm{D}}\right)$, where $\sigma_{\mathrm{j}}$ is the shear strength of the material, $\sigma$ is the adhesion stress. The straight line approximates the profile of the border between the regions of "grinding" and "breakaway" regimes of wear.

The numerically derived linear character of the dependence $\sigma_{\mathrm{D}}^{*}\left(\sigma_{\mathrm{j}}\right)$ well agrees with the following simple analytical estimation. When surfaces of unlinked elements come into mechanical contact, a chemical bonding appears between them (unlinked-to-linked transition of the state of the pair). In this case, the adhesion strength of the new linked pair of elements is assumed to be equal to the strength of intact material and the condition of local fracture is determined by fracture criterion (3).

The stress-strain state of the asperities near the contact patch can be characterized as «shear+compression». In these conditions breaking of link in a pair of elements in the contact patch or near (under) the contact patch doesn't lead to finishing of the interaction of elements since they are overlapped and remain in contact. Therefore these contact pairs again become linked. So, in the vicinity of the contact patch plastic shear dominated deformation of adjacent volume takes place while the material conserves its continuity. This deformation finally leads to smoothing of asperities as shown in Figure 3. The value of elastic strain energy per unit volume, which is necessary to accumulate in the contact patch to realize smoothing, can be estimated as $E_{\mathrm{el}}^{\text {strain }} \approx \sigma_{\mathrm{j}}^{2} / 2 G$.

A stress-strain state of the material in the region between asperity and the main part of the surface during loading is also complex and heterogeneous. In the left part of this region (Figure 2a) the stress state can be characterized as tension-dominated. Maximal tensile stress is concentrated in the vicinity of the corner between the asperity and the surface. In the right part of this region the stress state can be characterized as compression-dominated. So that the left part of boundary region characterized by tension-dominated stress state controls the formation of debris particle from the asperity. The condition of crack initiation in the boundary between the asperity and the main part of the surface layer (Figure 2b) is meeting the fracture criterion (3). After that, the diverging crack edges are attracted by adhesion stress. The attraction of the initiating crack edges close to each other by adhesive force is a necessary condition to provide healing of incipient crack and prevent crack propagation. The value of elastic strain energy per unit volume, which is necessary to supply to the boundary to form a tensile crack, can be estimated as $E_{\mathrm{el}}^{\text {adhesion }} \approx \sigma_{\mathrm{D}}^{2} / 2 E$.

The implementation of any of the modes of fracture of asperity ("breakaway" or "grinding") is determined by the relation of the energies $E_{\mathrm{el}}^{\text {adhesion }} / E_{\mathrm{el}}^{\text {strain }}$. Higher values of 
$E_{\mathrm{el}}^{\text {adhesion }}$ make the implementation of the "grinding" regime of wear of asperity more energetically favorable under constant values of other material parameters. When the relation $E_{\mathrm{el}}^{\mathrm{adhesion}} / E_{\mathrm{el}}^{\text {strain }}$ exceeds some critical value, a transition from "breakaway" to "grinding" mode takes place. Due to the fact that the relation $E_{\mathrm{el}}^{\text {adhesion }} / E_{\mathrm{el}}^{\text {strain }}$ is determined only by strength parameters (shear strength and adhesion stress) and elastic constants of a material, the critical value of the relation $E_{\mathrm{el}}^{\text {adhesion }} / E_{\mathrm{el}}^{\text {strain }}$ is a constant (assuming the loading conditions and other material parameters also remain constant). From that follows the linear dependence of the boundary (critical) value of adhesion stress on the shear strength of the material:

$$
\sigma_{\mathrm{D}}^{*}=B^{*}(1+v) \sigma_{\mathrm{j}}
$$

Here $B^{*}$ is a dimensionless parameter that depends both on the material parameters (except for shear strength and adhesion stress) and on the features of loading conditions and asperity geometry, which determine the features of stress distribution in the interacting asperities. The simulation results in Figure 5 are in a good agreement with the obtained simple analytical expression (5). The value of parameter $B^{*}$ is close to 1 in the considered case.

It is a well-known fact that a value of macroscopic shear stress of the most of materials depends on local mean stress [22]. This dependence is clearly manifested for materials with a limited ability for inelastic deformation, including composites, ceramics, rock materials, etc. [23]. Application of the two-parametric Drucker-Prager fracture criterion (1) allows taking into account this dependence in numerical simulations. Recall that in the given fracture criterion a linear proportionality of strength and local mean stress is postulated. A key dimensionless parameter of this criterion is a ratio of uniaxial compressive strength of the material to uniaxial tensile strength: $a=\sigma_{\mathrm{c}} / \sigma_{\mathrm{t}}$. It has a meaning of the sensitivity of the shear strength of the material to the local mean stress. We analyzed the influence of this parameter on the condition of the change of wear regime. In particular, we varied the value of $a$ having the value of $\sigma_{\mathrm{j}}$ constant (in accordance with (4), we varied both $\sigma_{\mathrm{c}}$ and $\sigma_{\mathrm{t}}$ to provide necessary variation of $a$ and keep constant value of $\sigma_{\mathrm{j}}$ ). We numerically derived the dependence of the threshold adhesion stress $\sigma_{\mathrm{D}}^{*}$ on the value of $a$ (Figure 6). It is seen form Figure 6 that the threshold adhesion stress separating the regions of "breakaway" and "grinding" regimes of wear is a decreasing function of $a$. Generally, the set of points corresponding to the different regimes of wear may be separated into three parts:

1.For $a \in[1,1.5]$ that corresponds to highly plastic materials, a "classic" transition of wear regime from "breakaway" to "grinding" takes place. At that, the separation line between two regions is a linearly decreasing function of $a$.

2.For $a \in(1.5,2.25]$ that corresponds to materials with a "limited" ductility, the "grinding" regime is not observed, and for relatively high values of the specific adhesion stress a transition from "breakaway" to "cleavage" regime occurs.

3. For $a>2.25$ that is typical for brittle materials, the destruction of asperities goes on in a "cleavage" regime only. 


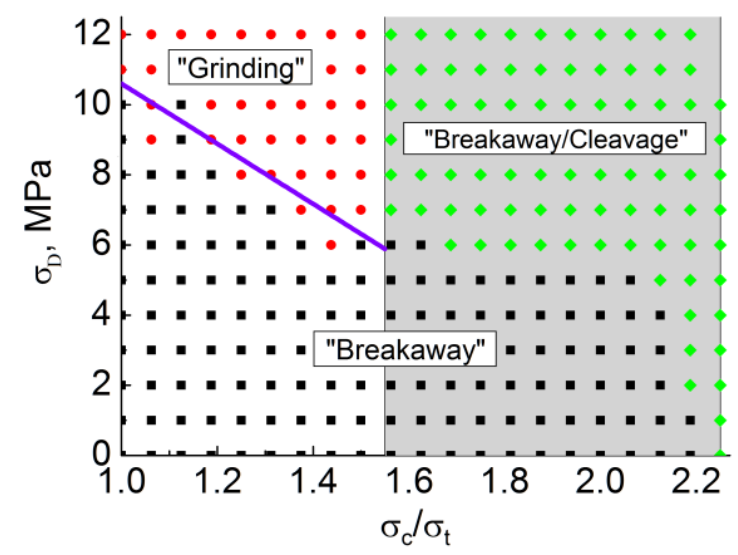

Fig. 6. The cloud of points corresponding to different regimes of wear under the variation of the specific adhesion stress $\sigma \mathrm{D}$ and the parameter $a$ of Drucker-Prager fracture criterion

The obtained results allow making a conclusion that the theory of Rabinowicz [12] gives reliable predictions of wear regime only for highly plastic metallic or polymeric materials with ductility based on a motion of translational defects of an atomic structure. Recall that the movability of translational defects depends on pressure only slightly. On the contrary, strength and, more generally, fracture of brittle materials and materials with a high initial concentration of damages significantly depend on a value of mean stress, that manifests in the prevalence of "cleavage" regime of wear (see Fig. 5). Hence, criterion (5) has to be extended in order to take into account the influence of hydrostatic pressure. We suggest the following empirical formulation of the criterion (5):

$$
\sigma_{\mathrm{D}}^{*} \approx \sigma_{\mathrm{j}} B_{0}(1-v)(2-a)
$$

where $B_{0}$ is the value of $B^{*}$ for highly ductile material ( $\left.a=1\right)$. Note that equation (6) includes the shear strength $\sigma_{\mathrm{j}}$ as well as the parameter $a=\sigma_{\mathrm{c}} / \sigma_{\mathrm{t}}$. Equation (6) allows describing the "separation line" between the "breakaway" and "grinding" regimes of wear in a wide range of values of material parameters.

\section{Conclusions}

We developed and verified a discrete element model that allows taking into account adhesive force acting between non-contacting discrete elements and "cold welding" effect. We carried out a numerical analysis of the applicability of Rabinowicz analytical criterion for prediction of wear regime for materials with non-ideal plasticity and pressure-dependent shear stress. The results of the analysis have shown the applicability of the given criterion for ductile materials having a weak dependence of shear strength on local mean stress $(a \sim 1)$. On the contrary, the conditions of the wear regime change for brittle materials (or for materials with a high initial concentration of failures), which have a pronounced dependence of shear stress on pressure $(a>1)$, significantly differ from the analytical estimation. Based on the obtained results we proposed a generalization of Rabinowicz criterion and found the intervals of values of mechanical properties of materials where the given generalized criterion allows obtaining reliable estimations. 
The work was carried out in the framework of the Fundamental Research Program of the State Academies of Sciences for 2013-2020 (Priority direction III.23).

\section{References}

1. P.L. Hurricks Wear, 26, 285 (1973)

2. Q. Zhang, J. Gu, S. Wei, M.Qi, Materials, 12, 1749 (2019)

3. R. Aghababaei, D. H. Warner, J.-F. Molinari, Proc. Natl. Acad. Sci. U.S.A., 114, 7935 (2017)

4. A. R. Warmuth, P. H. Shipway, W. Sun, Proc. R. Soc. A 471, 20140291 (2015)

5. N.K. Myshkin, D.V. Tkachuk, Polymer Tribology Fundamentals, In: Wang Q.J., Chung YW. (eds) Encyclopedia of Tribology (Springer, Boston, 2013)

6. M. Ciavarella, A. Papangelo, Phys. Mesomech. 21, 59 (2018)

7. R. Budakian, S. J. Putterman, Phys. Rev. B, 65, 235429 (2002)

8. D. V. Wagle, G. A. Baker, Mater. Horiz., 2, 157 (2015)

9. H. C. Meng, K. C. Ludema, Wear 181-183, 443 (1995).

10. L. Frérot, R. Aghababaei, J.-F. Molinari, J. Mech. Phys. Solids 114, 172 (2018).

11. J.-F. Molinari, R. Aghababaei, T. Brink, L. Frérot, and E. Milanese, Friction 6, 245 (2018).

12. E. Rabinowicz, Wear 2, 4 (1958).

13. E. Rabinowicz, Wear 7, 9 (1964)

14. E. Rabinowicz, Friction and Wear of Materials, 2nd ed. (Wiley, New York, 1995).

15. R. Holm, Electric Contacts: Theory and Application, 3rd ed. (Springer, Berlin, 1967)

16. R. Aghababaei, D. H.Warner, J.-F. Molinari, Nat. Commun. 7, 11816 (2016)

17. S. G. Psakhie, A. V. Dimaki, E. V. Shilko, S. V. Astafurov, Int. J. Num. Meth. Engng., 106, 623 (2016)

18. S. G. Psakhie, E. V. Shilko, A. S. Grigoriev, S. V. Astafurov, A. V. Dimaki, A. Yu. Smolin, Engng. Fract. Mech., 130, 96 (2014)

19. E. V. Shilko, S. G. Psakhie, S. Schmauder, V. L. Popov, S. V. Astafurov, A. Yu. Smolin, Comp. Mater. Sci., 102, 267 (2015)

20. S. Psakhie, E. Shilko, A. Smolin, S. Astafurov, V. Ovcharenko, Fract. Struct. Integr., 24, 59 (2013)

21. D. S. Dugdale, J. Mech. Phys. Solids, 8, 100 (1960)

22. D. C. Drucker, W. Prager, Quart. Appl. Math., 10. 157 (1952)

23. T. L. Anderson, Fracture Mechanics: Fundamentals and Applications, 3rd ed. (CRC Press: NY, 2005) 